\title{
Promoter hypermethylation influences the suppressive role of long non-coding RNA MEG3 in the development of multiple myeloma
}

\author{
WENJUN YU ${ }^{1,2^{*}}$, QINGLIN SHI ${ }^{2 *}$, CHAO WU $^{2}$, XUXING SHEN $^{2}$, LIJUAN CHEN $^{2 *}$ and JIAREN XU ${ }^{1 *}$ \\ ${ }^{1}$ Department of Geriatric Medicine, Geriatric Hospital of Nanjing Medical University, Jiangsu Province Geriatric Institute; \\ ${ }^{2}$ Department of Hematology, First Affiliated Hospital of Nanjing Medical University, Jiangsu Province Hospital, \\ Nanjing, Jiangsu 210000, P.R. China
}

Received January 12, 2019; Accepted December 31, 2019

DOI: $10.3892 /$ etm.2020.8723

\begin{abstract}
Methylation is a fundamental regulator of gene transcription. Long non-coding RNA maternally expressed 3 (MEG3) inhibits cell proliferation in various types of cancer. However, the molecular mechanisms of MEG3 methylation in the regulation of multiple myeloma (MM) are unknown. In the present study, MEG3 upregulation was negatively associated with the International Staging System (ISS) status of the bone marrow samples of 39 patients with MM. MEG3 overexpression in an MM cell line resulted in elevated p53 expression. Furthermore, the results of methylation-specific PCR revealed that the abnormal methylation status of the MEG3 promoter region was present in eight of the 39 bone marrow samples collected. Treatment of the MM cell line with the DNA methylation inhibitor 5-Aza-2'-deoxycytidine (5-Aza-CdR) resulted in tumor cell proliferation inhibition, apoptosis induction and $\mathrm{G}_{0} / \mathrm{G}_{1}$ cell cycle arrest. Furthermore, 5-Aza-CdR decreased aberrant hypermethylation of the MEG3 promoter and increased the expression of MEG3. However, 5-Aza-CdR exerted no effect on p53 expression. To the best of our knowledge, the present study is the first to report that the demethylation reagent 5-Aza-CdR may serve as a therapeutic agent in MM by upregulating MEG3 expression. However, the mechanism of action was independent of p53 expression.
\end{abstract}

Correspondence to: Dr Lijuan Chen, Department of Hematology, First Affiliated Hospital of Nanjing Medical University, Jiangsu Province Hospital, 300 Guangzhou Road, Nanjing, Jiangsu 210000, P.R. China

E-mail: chenljb@126.com

Dr Jiaren Xu, Department of Geriatric Medicine, Geriatric Hospital of Nanjing Medical University, Jiangsu Province Geriatric Institute, 18 Luojia Road, Nanjing, Jiangsu 210000, P.R. China

E-mail: xujiarencn@sina.com

*Contributed equally

Key words: long non-coding RNA, maternally expressed 3, promoter hypermethylation, multiple myeloma

\section{Introduction}

Multiple myeloma (MM) is one of the most common types of haematological cancer, which is becoming more common in the ageing population. It is also the cause of a number of fatal outcomes (1). As a haematological cancer that originates from the malignant transformation of plasma cells, MM maintains pathophysiologic heterogeneity due to its complex genetic background (2). Several distinct clinical phases of MM have been identified, including monoclonal gammopathy of undetermined significance and smoldering multiple myeloma (3). With the development and progression of MM, several distinct patterns of genetic aberration are recognized, including cytogenetic abnormalities, chromosomal aberration and signaling pathway disorders (4).

DNA methylation, a form of epigenetic control of gene transcription, refers to cytosine methylation at position 5 in the pyrimidine ring, which can result in inappropriate silencing of genes involved in diverse biological processes, including cell proliferation, apoptosis, migration and cell cycle arrest (5). In normal cells, unmethylated $\mathrm{CpG}$ islands (a cluster of CpG dinucleotides) are usually observed; however, human malignancies are characterized by the gain of methylation at promoter associated CpG islands (6). The role of DNA methylation in the mediation of multiple tumor suppressor gene and microRNA silencing has been implicated in the development and progression of MM (7).

Long non-coding RNAs (lncRNAs) are a class of non-coding RNA with a length of $>200$ nucleotides, which possess little to no capacity for protein synthesis (8). Numerous studies have reported that lncRNAs are deregulated in various types of cancer and are implicated in carcinogenesis and antitumor pathways (9). Wang et al (10) reported that protein tyrosine phosphatase L1 could be epigenetically regulated in MM; a process which can be reversed by 5 -Aza-2'-deoxycytidine (5-Aza-CdR), suggesting a potential therapeutic agent for MM. The maternally expressed 3 (MEG3) imprinted gene is located on chromosome 14q32, which produces a non-coding RNA transcript (11). IncRNA MEG3 has been identified as a tumor suppressor in various types of cancer, including meningioma (12), breast cancer (13), bladder cancer (14) and hepatocellular carcinoma (15). A previous study also demonstrated the anticancer effect of MEG3 in MM (16). 
The promoter region of MEG3 is rich in $\mathrm{CpG}$ islands, and the specific methylated and unmethylated $\mathrm{CpG}$ islands of differentially methylated regions (DMRs) are located upstream of the MEG3 gene (IG-DMR and MEG3-DMR) $(17,18)$. Furthermore, lncRNA MEG3 expression has been reported to be induced following epigenetic modification of DNA methylation in diverse malignancies, including gliomas (19), ovarian cancer $(20)$ and leukemia $(21,22)$.

In MM, Benetatos et al (21) observed MEG3 promoter hypermethylation in both bone marrow and peripheral blood samples. This hypermethylation was correlated with MM stage and subtype. Therefore, it was hypothesized that MEG3 expression could be epigenetically controlled by MEG3 promoter hypermethylation, which may ultimately influence the biological behavior of MM.

\section{Materials and methods}

Study subjects. The present study was approved by the Institutional Review Board of the First Affiliated Hospital of Nanjing Medical University. All participants provided written informed consent. Bone marrow biopsy samples were collected from 39 patients with newly diagnosed MM who were admitted to the First Affiliated Hospital of Nanjing Medical University between January 2009 and May 2014. Patient information is listed in Table I. The diagnosis of MM was established according to the standard morphological and immunophenotypical criteria (23). The subtype of MM was classified according to the monoclonal component. The stage of MM was classified according to the Durie-Salmon staging system and the International Staging System (ISS) (24).

MM cells were isolated from bone marrow samples using CD138 microbeads and MS-columns (Miltenyi Biotec; cat. no. 130-051-301) according to the manufacter's protocol. The MM cell line, ARP1 (American Type Culture Collection), was cultured in RPMI 1640 medium (Gibco; Thermo Fisher Scientific, Inc.) supplemented with $10 \%$ fetal bovine serum (Gibco; Thermo Fisher Scientific, Inc.) and 1\% penicillin-streptomycin in an incubator at $37^{\circ} \mathrm{C}$ with $5 \% \mathrm{CO}_{2}$.

$R N A$ isolation and reverse transcription-quantitative $P C R$ $(R T-q P C R)$. Total RNA was isolated from ARP1 cells and patient derived MM cells using TRIzol ${ }^{\circledR}$ reagent (Thermo Fisher Scientific, Inc.), according to the manufacturer's protcol. Total RNA was reverse transcribed to cDNA using the Primescipt RT Reagent kit with gDNA Eraser (Takara Biotechnology Co., Ltd.), according to the manufacturer's protocol.

qPCR was subsequently performed on a StepOne Plus ${ }^{\mathrm{TM}}$ Real-Time PCR system (Applied Biosystems; Thermo Fisher Scientific, Inc.) using a SYBR Green qRT-PCR assay according to the manufacturer's protocol (Takara Biotechnology Co., Ltd.). The following primer pairs, designed by Primer Premier 5 (Premier Biosoft International), were used for qPCR: MEG3 forward, 5'-GGAGCTGTTGAGCCTTCAGT-3' and reverse, 5'-CAAGCCCTGTGCTTTGGAAC-3'; and $\beta$-actin forward, 5'-AGCGAGCATCCCCCAAAGTT-3' and reverse, 5'-GGG CACGAAGGCTCATCATT-3'. The following thermocycling conditions were used for the qPCR: 40 cycles of denaturation at $95^{\circ} \mathrm{C}$ for $5 \mathrm{sec}$, annealing at $60^{\circ} \mathrm{C}$ for $30 \mathrm{sec}$, followed by a final extension at $72^{\circ} \mathrm{C}$ for $5 \mathrm{~min}$. MEG3 mRNA levels were quantified according to the standard curve of MEG3 and $\beta$-actin using the $2^{-\Delta \Delta C q}$ method (24). $\beta$-actin was used as the internal reference gene.

DNA isolation and methylation-specific PCR (MSP). Genomic (g)DNA was extracted from ARP1 cells and patient derived MM cells using a TIANamp Genomic DNA kit according to the manufacturer's protocol (Tiangen Biotech Co., Ltd.). Subsequently, DNA bisulfite conversion was performed on the gDNA using the EpiTect Plus Bisulfite kit (Qiagen GmbH), according to the manufacturer's protocol.

The methylation status of MEG3 was determined by MSP, using a Veriti96 PCR thermocycler (Applied Biosystems; Thermo Fisher Scientific, Inc.) with Taq PCR MasterMix (Tiangen Biotech Co., Ltd.). The following primers obtained from previous studies $(25,26)$ were used for MSP: Methylated primer pair forward, 5'-GTTAGTAATCGGGTTTGTCGGC-3' and reverse, 5'-AATCATAACTCCGAACACCCGCG-3'; and unmethylated primer pair forward, 5'-GAGGATGGTTAGTTA TTGGGGT-3' and reverse, 5'-CCACCATAACCAACACCC TATAATCACA-3'. PCR was performed using the following thermocycling conditions: $94^{\circ} \mathrm{C}$ for $3 \mathrm{~min} ; 5$ cycles of $94^{\circ} \mathrm{C}$ for $30 \mathrm{sec}, 70^{\circ} \mathrm{C}$ for $30 \mathrm{sec}$ and $72^{\circ} \mathrm{C}$ for $30 \mathrm{sec} ; 5$ cycles of $94^{\circ} \mathrm{C}$ for $30 \mathrm{sec}, 65^{\circ} \mathrm{C}$ for $30 \mathrm{sec}$ and $72^{\circ} \mathrm{C}$ for $30 \mathrm{sec} ; 30$ cycles of $94^{\circ} \mathrm{C}$ for $30 \mathrm{sec}, 60^{\circ} \mathrm{C}$ for $30 \mathrm{sec}$ and $72^{\circ} \mathrm{C}$ for $30 \mathrm{sec}$; and a final extension at $72^{\circ} \mathrm{C}$ for $7 \mathrm{~min}$. The PCR products were run on a $3 \%$ agarose gel and were subsequently identified by ethidium bromide staining at room temperature (27). The $\mathrm{CpG}$ island usually localizes to the DMR which is located within $\sim 4 \mathrm{~kb}$ of the DMR that contains the promoter of the MEG3 gene $(28,29)$. A $160 \mathrm{bp}$ product represented the methylated state and a $120 \mathrm{bp}$ product represented the unmethylated state of MEG3 (26).

Cell transfection and 5-Aza-CdR treatment. ARP-1 cells were cultured in DMEM medium (Gibco; Thermo Fisher Scientific, Inc.) supplemented with $10 \%$ fetal bovine serum (Gibco; Thermo Fisher Scientific, Inc.) and $1 \%$ penicillin-streptomycin in an incubator at $37^{\circ} \mathrm{C}$ with $5 \% \mathrm{CO}_{2}$. ARP1 cells $\left(2.0 \times 10^{6} /\right.$ well $)$ were plated into 6 -well plates and transfected with the $4 \mu \mathrm{g}$ pcDNA3.1-MEG3 or $4 \mu \mathrm{g}$ pcDNA3.1-empty (provided by Professor Wei De, Nanjing Medical University) using Lipofectamine ${ }^{\circledR} 2000$ (Thermo Fisher Scientific, Inc.), according to the manufacturer's protocol. For MEG3 knockdown, the following small interfering (si)RNA sequences were used for Lipofectamine ${ }^{\circledR} 2000$ transfection: si-MEG3, 5'-GCUCAUACUUUGACUCUAUTT-3'; and si-negative control (NC), 5'-UUCUCCGAACGUGUCACGUTT-3'. Both sequences were designed and synthesized by Shanghai GenePharma Co., Ltd.

ARP1 cells were seeded at $2 \times 10^{4}$ cells/well in 96-well culture plates and incubated with DMEM $(200 \mu \mathrm{l})$ containing $0.1,1,5,10,50$ or $100 \mu \mathrm{g} / \mathrm{ml} 5$-Aza-CdR (Sigma-Aldrich; Merck KGaA) for $72 \mathrm{~h}$ at $37^{\circ} \mathrm{C}$. Control cells were incubated with DMEM containing PBS $(20 \mu \mathrm{l})$. The Cell Counting Kit-8 (CCK-8) assay (Selleck Chemicals) was used to analyze cell proliferation according to the manufacturer's protocol. RT-qPCR and MSP were performed to assess the expression 
Table I. Distribution of variables of patients with multiple myeloma.

\begin{tabular}{|c|c|}
\hline Variable & Number $(\%)$ \\
\hline Median age (range) & $61(36-82)$ \\
\hline \multicolumn{2}{|l|}{ Sex } \\
\hline Male & $26(66.7)$ \\
\hline Female & $13(33.3)$ \\
\hline \multicolumn{2}{|l|}{ Subtypes } \\
\hline $\operatorname{IgG}$ & $18(46.2)$ \\
\hline $\operatorname{IgA}$ & $11(28.2)$ \\
\hline Light chain & $10(25.6)$ \\
\hline \multicolumn{2}{|l|}{ Durie-Salmon stage } \\
\hline I & $4(10.3)$ \\
\hline II & $5(12.8)$ \\
\hline III & $30(76.9)$ \\
\hline \multicolumn{2}{|c|}{ International Staging System stage } \\
\hline I & $5(12.8)$ \\
\hline II & $14(35.9)$ \\
\hline III & $20(51.3)$ \\
\hline \multicolumn{2}{|c|}{ Serum creatinine $(\mu \mathrm{mol} / \mathrm{l})$} \\
\hline$>176.8$ & 22 \\
\hline$\leq 176.8$ & 12 \\
\hline \multicolumn{2}{|c|}{ Serum calcium (mmol/l) } \\
\hline$>2.98$ & 31 \\
\hline$\leq 2.98$ & 3 \\
\hline N/A & 5 \\
\hline
\end{tabular}

N/A, not detected.

of MEG3 mRNA and the methylation status of the MEG3 promoter, respectively.

For restoration experiments, ARP1 cells were treated with 5-Aza-CdR $\left(50 \mu \mathrm{g} / \mathrm{ml}\right.$ for $48 \mathrm{~h}$ at $\left.37^{\circ} \mathrm{C}\right)$, followed by MEG3 knockdown. MEG3 expression was detected by RT-qPCR at $48 \mathrm{~h}$ post-transfection. ARP1 cell proliferation following MEG3 knockdown was analyzed by the CCK-8 assay at 0, 24, 48 and $72 \mathrm{~h}$ post-transfection.

Western blotting. ARP1 cells and patient derived MM cells were lysed and total protein was extracted using RIPA buffer (Beyotime Institute of Biotechnology), according to the manufacturer's protocol. Total protein was quantified using the Bicinchoninic Acid Protein Assay kit (Beyotime Institute of Biotechnology), according to the manufacturer's protocol. Subsequently, $50 \mu \mathrm{g}$ protein/lane was separated on $10 \%$ SDS-PAGE gels by electrophoresis and then transferred to PVDF membranes. The membranes were blocked with $5 \%$ skimmed milk for $\sim 2 \mathrm{~h}$ at room temperature and then incubated with anti-p53 (1:1,000; Cell Signaling Technology, Inc. cat. no. 2524) and anti-GAPDH (1:1,000; Cell Signaling Technology, Inc.; cat. no. 5174) primary antibodies overnight at $4^{\circ} \mathrm{C}$. Membranes were washed for $1 \mathrm{~h}$ with TBST buffer. Following the primary incubation, membranes were incubated for $1.5 \mathrm{~h}$ with appropriate secondary antibodies (horseradish peroxidase conjugated goat anti-rabbit IgG H\&L; 1:4,000; Abcam; cat. no. ab6721) at room temperature. Protein bands were visualized using the Chemiluminescence horseradish peroxidase substrate (cat. no. P90720; EMD Millipore) and the Molecular Imager ChemiDoc XRS+ chemiluminescence system (Bio-Rad Laboratories, Inc.). Protein expression was quantified using Image Lab software version 5.0 (Bio-Rad Laboratories, Inc.) with GAPDH as the loading control.

Flow cytometry. To analyze the cell cycle, ARP1 cells $\left(2.0 \times 10^{6} /\right.$ well $)$ were plated in 6-well plates and treated with a series of concentrations of 5-Aza-CdR $(0,5,10$ and $50 \mu \mathrm{g} / \mathrm{ml})$. After $48 \mathrm{~h}$ incubation at $37^{\circ} \mathrm{C}$ with $5 \% \mathrm{CO}_{2}$, cells were washed with PBS and fixed with $75 \%$ cold ethanol for $24 \mathrm{~h}$ at $-20^{\circ} \mathrm{C}$. Subsequently, the cells were washed with PBS and stained using the Cell Cycle Detection kit according to the manufacturer's protocol (Nanjing KeyGen Biotech Co., Ltd.) at room temperature for $30 \mathrm{~min}$ to analyze the cell cycle with FACS (Becton, Dickinson and Company).

To analyze apoptosis, ARP1 cells $\left(2.0 \times 10^{6} /\right.$ well) were plated in 6-well plates and treated with a series of concentrations of 5-Aza-CdR $(0,5,10$ and $50 \mu \mathrm{g} / \mathrm{ml})$. After $48 \mathrm{~h}$ treatment at $37^{\circ} \mathrm{C}$ with $5 \% \mathrm{CO}_{2}$, the cells were washed with PBS. Subsequently, the cells were harvested and stained using the Annexin V-FITC Apoptosis Detection kit (Nanjing KeyGen Biotech Co., Ltd.), according to the manufacturer's protocol. Cells were stained with Annexin V and PI at $4^{\circ} \mathrm{C}$ for $15 \mathrm{~min}$ in the dark, and subjected to FACS analysis (Becton, Dickinson and Company).

For restoration experiments, ARP1 cells were treated with 5-Aza-CdR $\left(50 \mu \mathrm{g} / \mathrm{ml}\right.$ for $48 \mathrm{~h}$ at $37^{\circ} \mathrm{C}$ with $\left.5 \% \mathrm{CO}_{2}\right)$, followed by MEG3 knockdown. At $48 \mathrm{~h}$ post-transfection, cells were used for cell cycle and apoptosis analyses.

Statistical analysis. The schematic diagram of $\mathrm{CpG}$ islands in the human MEG3 promoter was performed using MethPrimer software (Version 1.0; www.urogene.org/methprimer). Statistical analysis was performed using GraphPad Prism (version 5; GraphPad Software, Inc.). A chi-squared test was used to compare categorical variables. Data were presented as the mean \pm standard deviation from at least three repeats. Data were compared using a Student's t-test or one-way ANOVA followed by Tukey's post hoc test. $\mathrm{P}<0.05$ was considered to indicate a statistically significant difference.

\section{Results}

MEG3 expression is negatively associated with ISS stage in patients with newly diagnosed MM. In the present study, MEG3 levels in 39 newly diagnosed MM bone marrow samples were quantified via RT-qPCR. The clinical characteristics of the patients with MM are listed in Table I. Five representative samples were selected to present the methylation pattern of the MEG3 DMRs (Fig. 1A). The standard curve used to detect MEG3 and $\beta$-actin expression levels is presented in Fig. 1B. Of the 39 samples, MEG3 expression was detected in 36 and the remaining 3 exhibited an MEG3 expression below the limit of detection (Fig. 1C). MEG3 expression was also negatively associated with ISS stage in patients with newly diagnosed 
A

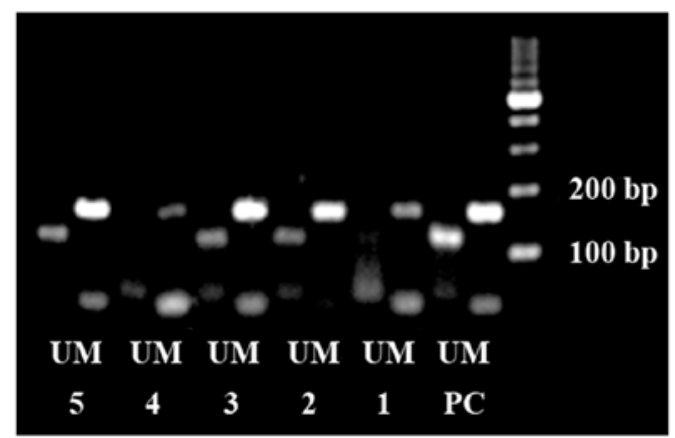

C

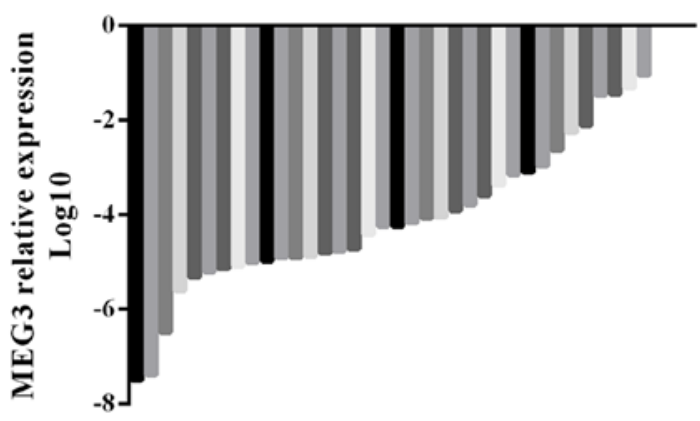

$\mathbf{E}$
B

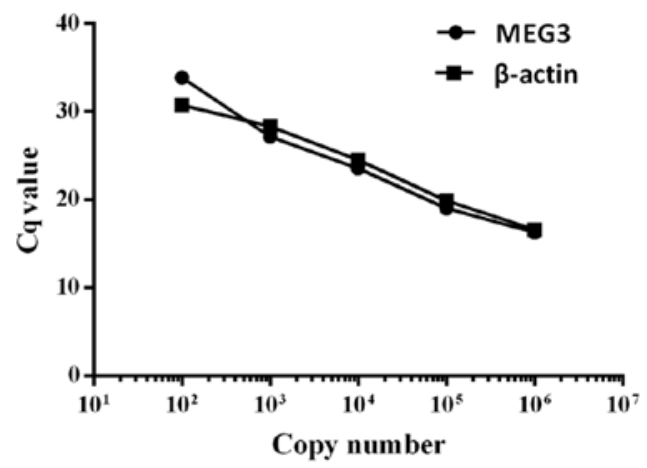

D

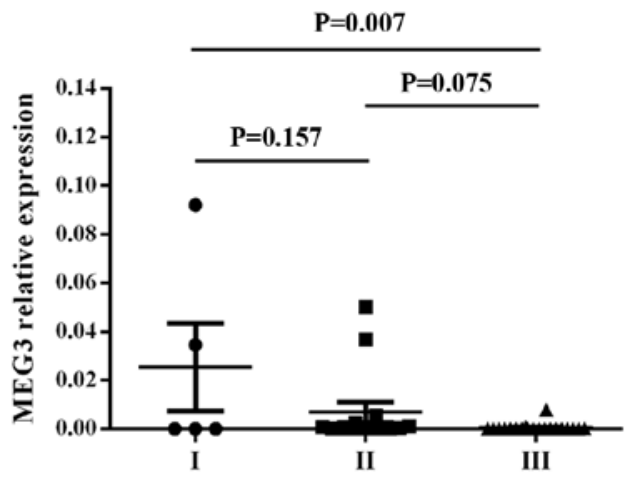

ISS Stage

MEG3 promoter analysis

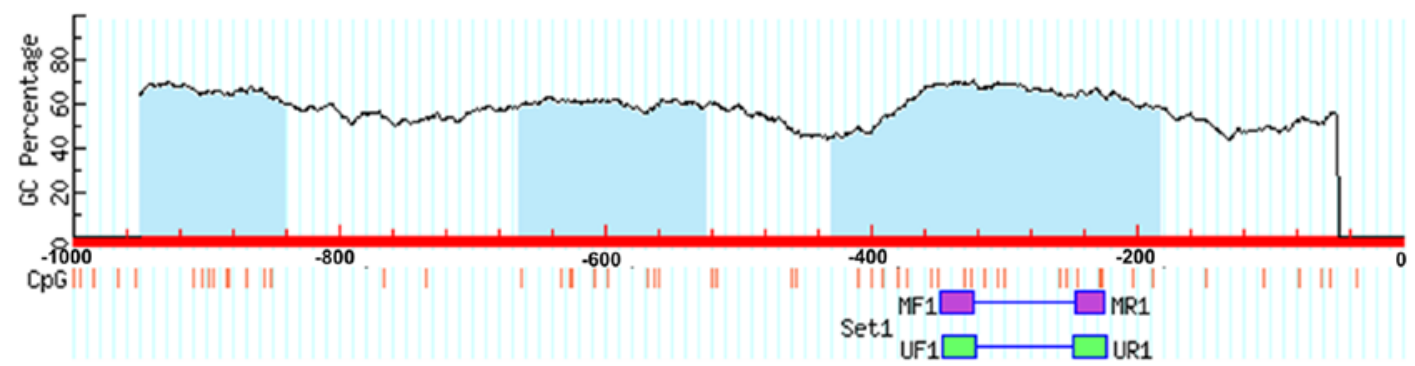

Figure 1. MEG3 methylation status and MEG3 expression in the bone marrow samples of patients with MM. (A) MEG3 methylation status. Samples 1 and 4 exhibited an abnormal methylation status. Samples 2, 3 and 5 exhibited a normal methylation status. (B) A standard curve was used to calculate the expression of MEG3 and $\beta$-actin following reverse transcription-quantitative PCR. (C) Relative expression of MEG3 in 39 bone marrow samples of patients with MM. MEG3 expression was detected in 36 samples. (D) MEG3 expression was negatively associated with International Staging System stage. (E) Schematic diagram of $\mathrm{CpG}$ islands (blue area) in the human MEG3 promoter, identified using MethPrimer software. The position of CpG islands in relation to the MEG3 transcription starting site and MSP primers were depicted. MEG3, maternally expressed 3; MM, multiple myeloma; PC, positive control; M, methylated band; $\mathrm{U}$, unmethylated band; MF1, methylated forward 1; MR1, methylated reverse 1; UF1, unmethylated forward 1; UR1, unmethylated reverse 1.

MM (Fig. 1D), indicating that MEG3 may serve as a tumor suppressor in human MM. The schematic diagram of $\mathrm{CpG}$ islands in the human MEG3 promoter is presented in Fig. 1E.

MEG3 methylation status and expression is restored after treatment with 5-Aza-CdR. In the 39 newly diagnosed MM samples, an abnormal methylation pattern of the MEG3 DMRs was identified in eight of the bone marrow samples. Chi-squared test was used to examine the association between MEG3 methylation status and ISS stage (Table II); however, a significant correlation was not observed. ARP1 cells were treated with different concentrations of 5-Aza-CdR $(0,5,10$ or $50 \mu \mathrm{g} / \mathrm{ml}$ ). Re-expression of MEG3 (Fig. 2B) and reversed abnormal methylation pattern of the MEG3 promoter (Fig. 2A) were observed following treatment with 5-Aza-CdR. These results indicated that $\mathrm{CpG}$ methylation may downregulate MEG3 mRNA expression in MM cells.

5-Aza-CdR contributes to the inhibition of MM cells. To investigate the role of 5-Aza-CdR in cell proliferation, ARP1 cells were treated with different concentrations of 5-Aza-CdR. The results of the CCK- 8 assay revealed that proliferation was inhibited by 5 -Aza-CdR in a dose-dependent manner (mean inhibition proportion of $0,5,10,50$ and $100 \mu \mathrm{g} / \mathrm{ml}$ for $24 \mathrm{~h}$ : 
Table II. Distribution of methylation status among the International Staging System stages in patients with multiple myeloma.

\begin{tabular}{lcrrr}
\hline & \multicolumn{3}{c}{$\begin{array}{c}\text { International staging } \\
\text { system stage }\end{array}$} & \\
\cline { 2 - 5 } Methylation status & I & II & III & P-value \\
\hline $\begin{array}{l}\text { Unmethylated } \\
\text { Methylated }\end{array}$ & $4(12.9)$ & $12(38.7)$ & $15(48.4)$ & 0.748 \\
\hline & $1(12.5)$ & $2(25.0)$ & $5(62.5)$ & \\
\hline
\end{tabular}

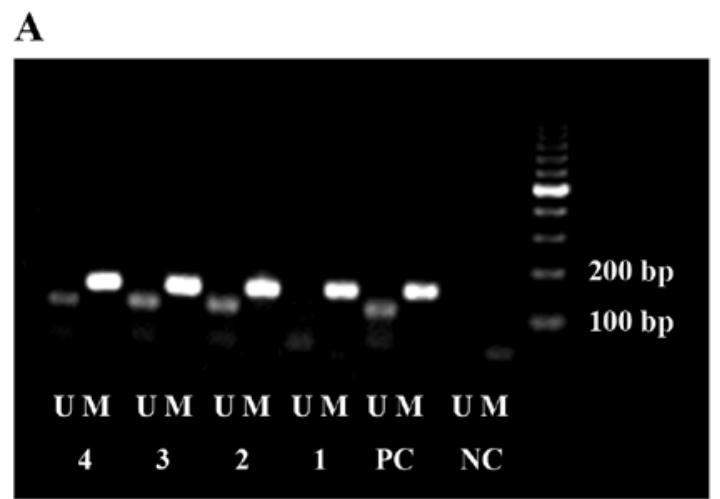

B

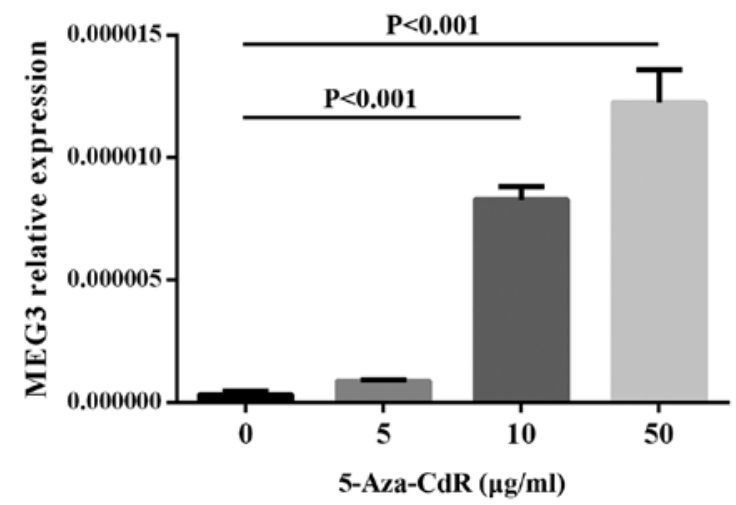

Figure 2. 5-Aza-CdR restores the normal MEG3 methylation status and MEG3 expression of ARP1 cells. (A) MEG3 methylation status and (B) expression of ARP1 cells. 5-Aza-CdR, 5-Aza-2'-deoxycytidine; MEG3, maternally expressed 3; NC, negative control; PC, positive control; $\mathrm{M}$, methylated band; U, unmethylated band; $1,0 \mu \mathrm{g} / \mathrm{ml}$ 5-Aza-CdR; $2,5 \mu \mathrm{g} / \mathrm{ml}$ 5-Aza-CdR; 3, $10 \mu \mathrm{g} / \mathrm{ml}$ 5-Aza-CdR; 4, $50 \mu \mathrm{g} / \mathrm{ml}$ 5-Aza-CdR.

3.0, 38.5, 42.8, 59.6 and 61.5\%; $48 \mathrm{h:} 7.3,45.3,53.9,74.3$ and $79.8 \%$; and 72 h: 6.0, 45.1, 69.5, 91.6 and 93.5\%; Fig. 3A). The results of the apoptosis assay revealed that the number of apoptotic cells increased by 5 -Aza-CdR in a dose-dependent manner $(6.02 \%$ for $0 \mu \mathrm{g} / \mathrm{ml}, 19.45 \%$ for $5 \mu \mathrm{g} / \mathrm{ml}, 24.58 \%$ for $10 \mu \mathrm{g} / \mathrm{ml}$, and $51.49 \%$ for $50 \mu \mathrm{g} / \mathrm{ml}$; Fig. 3B and D). Similarly, cell cycle analysis demonstrated that the number of ARP1 cells arrested at the $\mathrm{G}_{0} / \mathrm{G}_{1}$ phase was increased by $5-\mathrm{Aza}-\mathrm{CdR}$ in a dose-dependent manner $(27.30 \pm 0.74$ for $0 \mu \mathrm{g} / \mathrm{ml}, 31.93 \pm 0.79$ for $5 \mu \mathrm{g} / \mathrm{ml}, 43.27 \pm 1.02$ for $10 \mu \mathrm{g} / \mathrm{ml}$, and $48.00 \pm 0.36$ for $50 \mu \mathrm{g} / \mathrm{ml}$; Fig. $3 \mathrm{C}$ and E). The aforementioned results indicated that 5-Aza-CdR inhibits the proliferation of MM cells.
Association between MEG3 and p53 expression. The present study investigated whether MEG3 regulated the expression of p53. ARP1 cells were successfully transfected with pcDNA3.1-MEG3 or pcDNA3.1-empty (Fig. 4A). The pcDNA3.1-MEG3 group displayed significantly increased levels of p53 protein (Fig. 4B). Furthermore, whether 5-Aza-CdR influences the expression of p53 was investigated. The results revealed that 5-Aza-CdR treatment did not alter the p53 expression of ARP1 cells (Fig. 4C).

Restoration experiments. ARP1 cells treated with 5-Aza-CdR followed by MEG3 knockdown were used to investigate whether the tumor suppressive role of 5-Aza-CdR in MM cells may be due to the upregulation of MEG3. MEG3 expression levels were detected in untreated, si-NC, si-MEG3, 5-Aza-CdR, 5-Aza-CdR + si-NC and 5-Aza-CdR + si-MEG3 groups, which demonstrated that 5-Aza-CdR could upregulate MEG3 expression in ARP1 cells (Fig. 5A). In co-treated cells, the increased antiproliferation (Fig. 5B), proapoptotic (Fig. 5C and D) and enhanced cell cycle arrest (Fig. 5E and F) effects induced by 5-Aza-CdR treatment were partly inhibited by MEG3 knockdown.

\section{Discussion}

In the present study, low MEG3 expressions were associated with higher ISS stages. A previous study reported that MEG3 overexpression inhibited proliferation, promoted apoptosis and blocked the cell cycle in ARP1 cells (16). Furthermore, the demethylating agent, 5-Aza-CdR, reversed the hypermethylation status of the MEG3 promoter and increased MEG3 expression in ARP1 cells. Additionally, 5-Aza-CdR produced an antiproliferative effect on ARP1 cells, which was reversed by MEG3 knockdown. Taken together, the results of the present study suggested that MEG3 may serve as a tumor suppressor gene in MM and that MEG3 may be epigenetically modified by the hypermethylation status of MEG3 promoter.

MEG3 is a maternally expressed imprinted gene, which encodes an lncRNA (30). Multiple tumor samples have been tested for MEG3 expression and loss of MEG3 expression has been identified in the majority of tumor types, including bladder (14), epithelial ovarian (20) and gallbladder cancer (31), as well as glioma (19) and nasopharyngeal carcinoma (32). In MM, ISS stage is the most important prognostic system (23). In the present study, MEG3 expression was detected in bone marrow samples derived from patients with MM. Loss of MEG3 expression was associated with higher ISS stages, indicating that MEG3 may serve as a prognostic biomarker in MM. The tumor suppressor role of MEG3 in MM identified in the present study is consistent with the aforementioned role of MEG3 in other malignancies identified in previous studies.

The tumor suppressor function of p53 has long been recognized and p53 has been reported to be mutated, which may lead to the loss of wild-type p53 activity in the majority of human malignancies (33). p53 abnormalities are regarded as independent prognostic markers in MM $(34,35)$. Previously, a study reported that MEG3 could stimulate p53-dependent transcription (12). p53 exhibits a relative low expression level due to rapid degradation caused by the ubiquitin-proteasome pathway (36). The p53 protein 

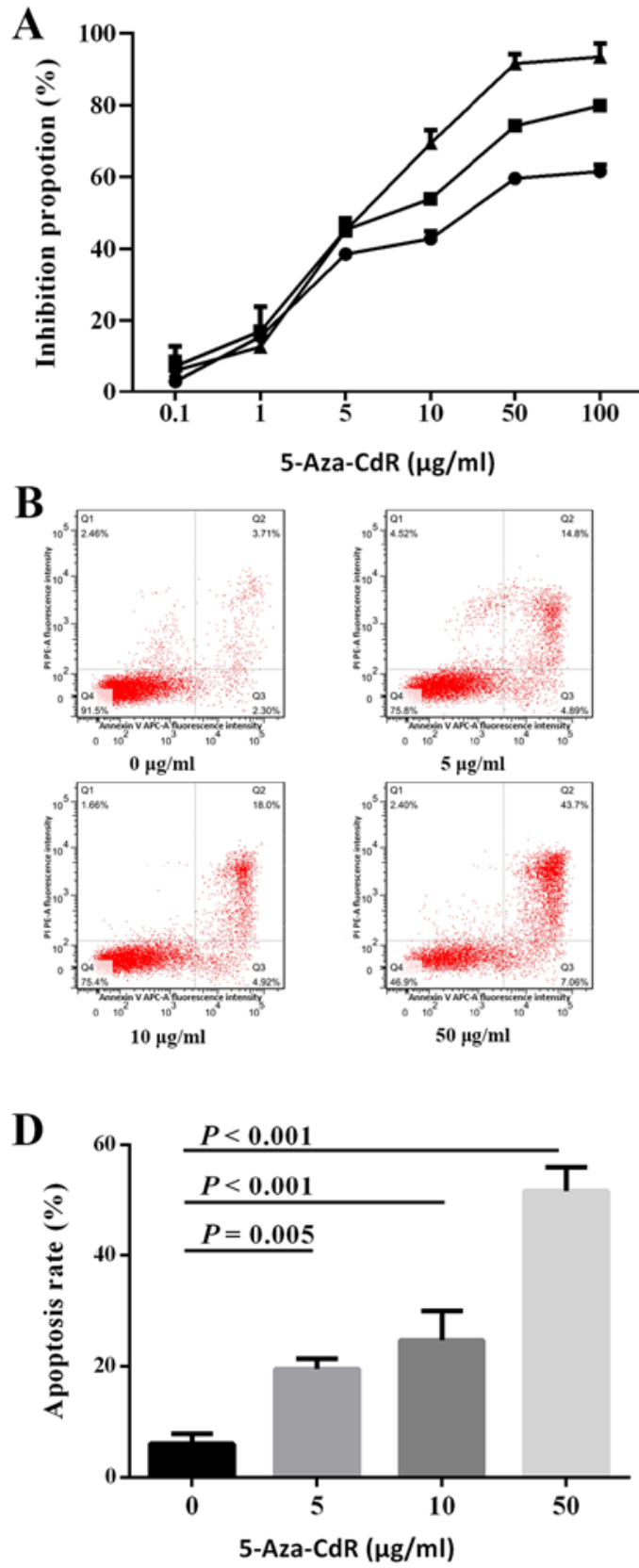

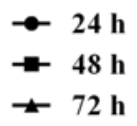

C
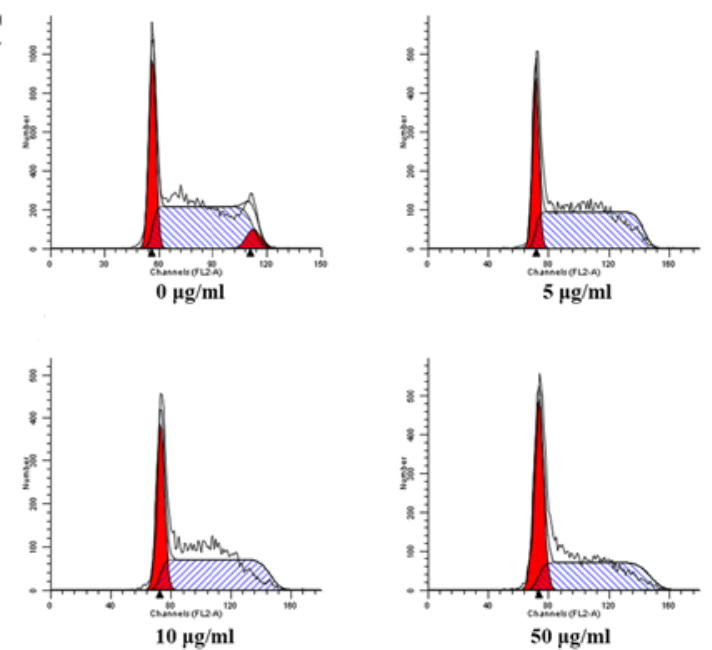

E

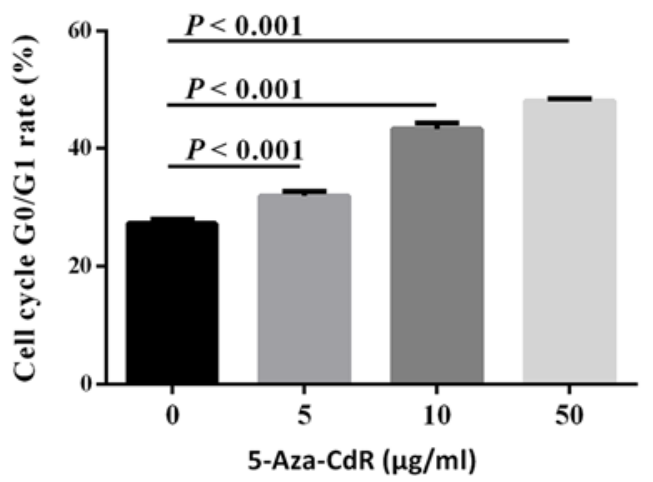

Figure 3. 5-Aza-CdR functions as an anti-tumor factor in the ARP1 cells. (A) 5-Aza-CdR inhibited the proliferation of the ARP1 cell line. (B) Representative images of the flow cytometry analysis of the (B) apoptosis and (C) cell cycle of ARP1 cells following 5-Aza-CdR treatment. (D) 5-Aza-CdR promoted the apoptosis of the ARP1 cell line. (E) 5-Aza-CdR arrested the cell cycle at $\mathrm{G}_{0} / \mathrm{G}_{1}$ in the ARP1 cell line. 5-Aza-CdR, 5-Aza-2'-deoxycytidine.

is regulated by MDM2 proto-oncogene (MDM2), which is an E3 ubiquitin ligase that inhibits the function of p53 and promotes its degradation (37). MEG3 has been identified as a tumor suppressor, which exerts its effect by downregulating MDM2 expression, as well as activating p53 (38). Furthermore, MDM2 suppression contributes to p53 accumulation induced by MEG3 (39). Previous studies have demonstrated that p53 serves as target of MEG3 in multiple types of cancer, including breast (13), non-small cell lung (38) and bladder cancer (40), as well as glioma (19) and hepatoma (41). In the present study, p53 protein levels were upregulated in ARP1 cells overexpressing MEG3, indicating that MEG3 may suppress MM cell proliferation by upregulating p53.
DNA methylation, an epigenetic regulation mechanism, serves a role in silencing MEG3 gene expression in different types of cancer (42). The role of the DNA methylation inhibitor 5-Aza-CdR is associated with the hypermethylation regulation of various genes in MM (10). Downregulation of MEG3 expression is associated with the hypermethylation status of the MEG3 promoter in a number of malignancies, including glioma (19), ovarian cancer (20) and leukemia (21). In MM, Benetatos et al (21) reported MEG3 promoter hypermethylation status in 12 out of 21 bone marrow samples and 9 out of 14 peripheral blood samples. In the present study, MEG3 promoter methylation status was identified in the bone marrow samples of 39 patients with MM and abnormal hypermethylation was identified in eight samples. The 
A

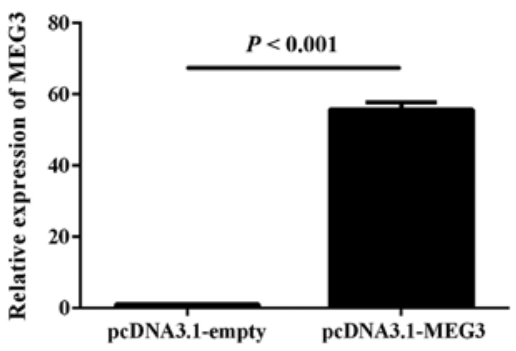

B
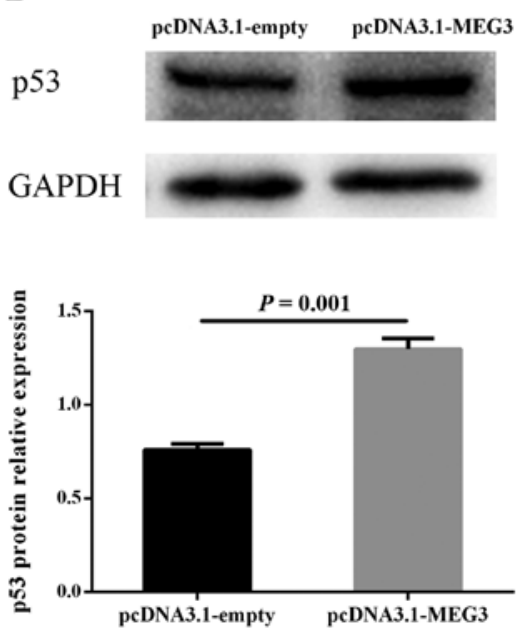

C 5 -Aza-CdR

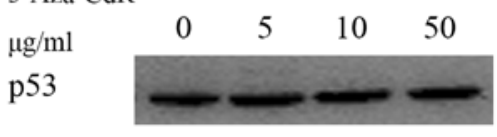

GAPDH

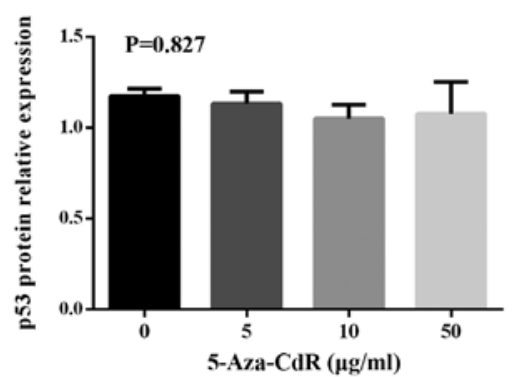

Figure 4. Effect of MEG3 overexpression and 5-Aza-CdR on p53 expression in ARP1 cells. (A) MEG3 overexpression efficiency in ARP1 cells transfected with pcDNA3.1-empty and pcDNA3.1-MEG3. (B) MEG3 overexpression increased the protein levels of p53 in the ARP1 cell line; (C) 5-Aza-CdR exhibited no effect on p53 protein expression. MEG3, maternally expressed 3; 5-Aza-CdR, 5-Aza-2'-deoxycytidine.

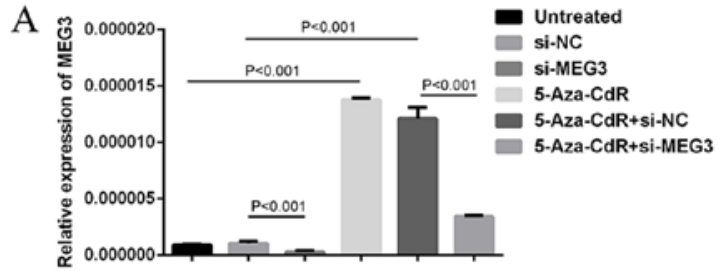

C

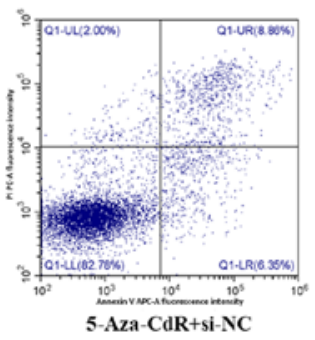

$\mathrm{E}$

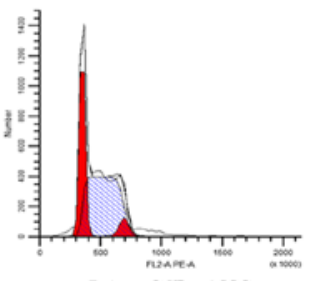

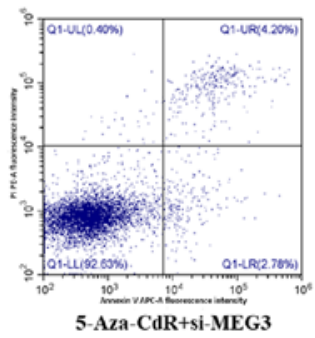

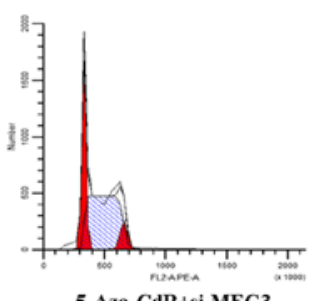

$\mathrm{B}$

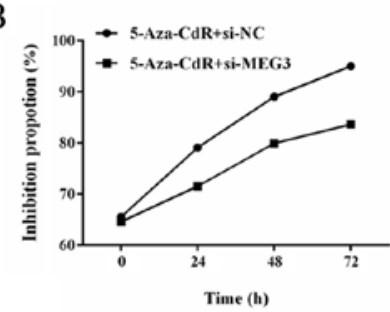

D
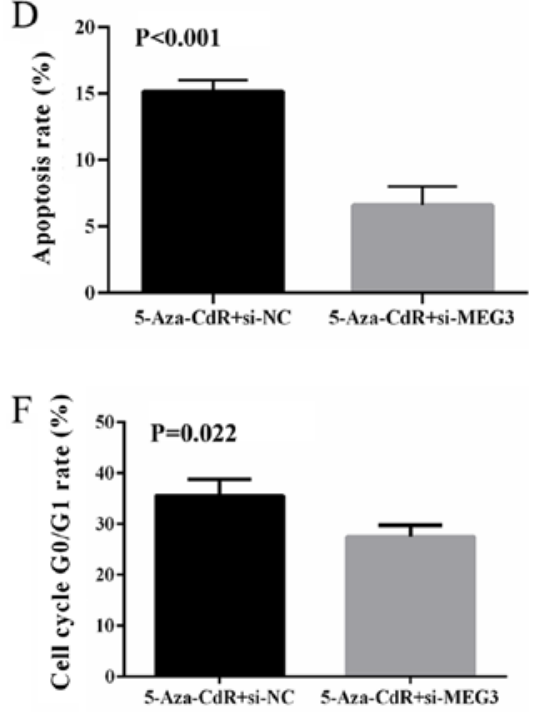

Figure 5. MEG3 knockdown reverses the antitumor effects of 5-Aza-CdR. Analysis of (A) MEG3 mRNA expression, (B) cell proliferation, (C) representative plots and (D) quantification of apoptosis. (E) Representative plots and (F) quantification of the cell cycle in ARP1 cells treated with 5-Aza-CdR followed by MEG3 knockdown. MEG3, maternally expressed 3; 5-Aza-CdR, 5-Aza-2'-deoxycytidine; si, small interfering RNA; NC, negative control. 
discrepancy between the present study and previous studies could be attributed to the small sample size and diverse genetic backgrounds of the patients included in the current study. Furthermore, no significant association was identified between MEG3 methylation status and ISS stage, which could be attributed to the small number of samples and the low proportion of samples with an abnormal methylation status. The functional role of 5-Aza-CdR in ARP1 cells was further explored. The results further demonstrated a demethylation effect of 5-Aza-CdR on MEG3 promoter hypermethylation in ARP1 cells. In addition, MEG3 levels revealed a dose-dependent relationship with 5-Aza-Cdr concentration. Furthermore, 5-Aza-CdR inhibited proliferation, promoted apoptosis and induced $G_{0} / G_{1}$ cell cycle arrest in ARP1 cells. The effects of 5-Aza-CdR were reversed by MEG3 knockdown. The results suggested that the demethylation reagent, 5-Aza-CdR, might restore MEG3 expression by demethylating the MEG3 promoter and therefore, may exert an anticancer effect in MM. Furthermore, the effect of 5-Aza-CdR on p53 expression was investigated; however, no alterations to the levels of p53 protein were observed. This may be attributed to the different effect of 5-Aza-CdR on different genes. Therefore, the results indicated that the antitumor effect of 5-Aza-CdR involved MEG3 but was independent of p53.

In conclusion, the present study suggested that MEG3 may serve as a tumor suppressor by upregulating p53 levels in MM. Furthermore 5-Aza-CdR inhibited MM cell proliferation by upregulating MEG3 expression. However, this was independent of p53 expression. Further investigation into how 5-Aza-CdR affects MEG3 and why p53 expression is not altered in $\mathrm{MM}$ is required. Additionally, further investigation into the mechanisms of MEG3 may provide novel therapeutic targets for MM.

\section{Acknowledgements}

The authors would like to thank Professor Wei De (Nanjing Medical University) for providing the pcDNA3.1-MEG3 and pcDNA3.1-empty plasmid.

\section{Funding}

The present study was supported by the National Natural Science Foundation of China (grant nos. 81800200 and 81670199) and the Jiangsu Province's Medical Elite Program (grant no. ZDRCA2016015).

\section{Availability of data and materials}

The datasets used and/or analyzed during the current study are available from the corresponding author on reasonable request.

\section{Authors' contributions}

WY and QS assisted with all experiments and wrote the manuscript. CW and XS were responsible for the flow cytometry experiments and statistical analysis. JX and LC designed the current study and critically reviewed the manuscript. All authors read and approved the final manuscript.

\section{Ethics approval and consent to participate}

The present study was approved by the Institutional Review Board of the First Affiliated Hospital of Nanjing Medical University. All participants provided written informed consent.

\section{Patient consent for publication}

Not applicable.

\section{Competing interests}

The authors declare that they have no competing interests.

\section{References}

1. Kazandjian D: Multiple myeloma epidemiology and survival: A unique malignancy. Semin Oncol 43: 676-681, 2016.

2. Morgan GJ, Walker BA and Davies FE: The genetic architecture of multiple myeloma. Nat Rev Cancer 12: 335-348, 2012.

3. Mateos MV and Landgren O: MGUS and smoldering multiple myeloma: Diagnosis and epidemiology. Cancer Treat Res 169: 3-12, 2016.

4. Glavey SV, Manier S, Sacco A, Salem K, Kawano Y, Bouyssou J, Ghobrial IM and Roccaro AM: Epigenetics in multiple myeloma. Cancer Treat Res 169: 35-49, 2016.

5. Wilkins JF: Genomic imprinting and methylation: Epigenetic canalization and conflict. Trends Genet 21: 356-365, 2005.

6. Liyanage VR, Jarmasz JS, Murugeshan N, Del Bigio MR, Rastegar M and Davie JR: DNA modifications: Function and applications in normal and disease States. Biology (Basel) 3: 670-723, 2014.

7. Wong KY and Chim CS: DNA methylation of tumor suppressor protein-coding and non-coding genes in multiple myeloma. Epigenomics 7: 985-1001, 2015.

8. Wilusz JE, Sunwoo H and Spector DL: Long noncoding RNAs: Functional surprises from the RNA world. Genes Dev 23: 1494-1504, 2009.

9. Schmitt AM and Chang HY: Long noncoding RNAs in cancer pathways. Cancer Cell 29: 452-463, 2016.

10. Wang W, Wang J, Chen M, Liang Y, LI Z, Zhang Z and Jing H: 5-Azacitidine remolds the methylation status and inhibits growth in multiple myeloma. Blood 126: 4817, 2015.

11. Miyoshi N, Wagatsuma H, Wakana S, Shiroishi T, Nomura M, Aisaka K, Kohda T, Surani MA, Kaneko-Ishino T and Ishino F: Identification of an imprinted gene, Meg3/Gt12 and its human homologue MEG3, first mapped on mouse distal chromosome 12 and human chromosome 14q. Genes Cells 5: 211-220, 2000.

12. Zhang X, Gejman R, Mahta A, Zhong Y, Rice KA, Zhou Y, Cheunsuchon P, Louis DN and Klibanski A: Maternally expressed gene 3, an imprinted noncoding RNA gene, is associated with meningioma pathogenesis and progression. Cancer Res 70: 2350-2358, 2010.

13. Sun L, Li Y and Yang B: Downregulated long non-coding RNA MEG3 in breast cancer regulates proliferation, migration and invasion by depending on p53's transcriptional activity. Biochem Biophys Res Commun 478: 323-329, 2016.

14. Ying L, Huang Y, Chen H, Wang Y, Xia L, Chen Y, Liu Y and Qiu F: Downregulated MEG3 activates autophagy and increases cell proliferation in bladder cancer. Mol Biosyst 9: 407-411, 2013.

15. Zhuo H, Tang J, Lin Z, Jiang R, Zhang X, Ji J, Wang P and Sun B: The aberrant expression of MEG3 regulated by UHRF1 predicts the prognosis of hepatocellular carcinoma. Mol Carcinog 55: 209-219, 2016.

16. Shen X, Bai H, Zhu H, Yan Q, Yang Y, Yu W, Shi Q, Wang J, Li $J$ and Chen L: Long non-coding RNA MEG3 functions as a competing endogenous RNA to regulate HOXA11 expression by sponging miR-181a in multiple myeloma. Cell Physiol Biochem 49: 87-100, 2018.

17. Astuti D, LatifF, Wagner K, Gentle D, Cooper WN, Catchpoole D, Grundy R, Ferguson-Smith AC and Maher ER: Epigenetic alteration at the DLK1-GTL2 imprinted domain in human neoplasia: Analysis of neuroblastoma, phaeochromocytoma and Wilms tumour. Br J Cancer 92: 1574-1580, 2005. 
18. Kagami M, O'Sullivan MJ, Green AJ, Watabe Y, Arisaka O, Masawa N, Matsuoka K, Fukami M, Matsubara K, Kato F, et al: The IG-DMR and the MEG3-DMR at human chromosome 14q32.2: Hierarchical interaction and distinct functional properties as imprinting control centers. PLoS Genet 6: e1000992, 2010

19. Li J, Bian EB, He XJ, Ma CC, Zong G, Wang HL and Zhao B Epigenetic repression of long non-coding RNA MEG3 mediated by DNMT1 represses the p53 pathway in gliomas. Int J Oncol 48 : 723-733, 2016

20. Sheng X, Li J, Yang L, Chen Z, Zhao Q, Tan L, Zhou Y and Li J: Promoter hypermethylation influences the suppressive role of maternally expressed 3, a long non-coding RNA, in the development of epithelial ovarian cancer. Oncol Rep 32: 277-285, 2014.

21. Benetatos L, Hatzimichael E, Dasoula A, Dranitsaris G, Tsiara S, Syrrou M, Georgiou I and Bourantas KL: CpG methylation analysis of the MEG3 and SNRPN imprinted genes in acute myeloid leukemia and myelodysplastic syndromes. Leuk Res 34 148-153, 2010.

22. Li ZY, Yang L, Liu XJ, Wang XZ, Pan YX and Luo JM: The long noncoding RNA MEG3 and its target miR-147 regulate JAK/STAT pathway in advanced chronic myeloid leukemia. EBioMedicine 34: 61-75, 2018.

23. Greipp PR, San Miguel J, Durie BG, Crowley JJ, Barlogie B, Bladé J, Boccadoro M, Child JA, Avet-Loiseau H, Kyle RA, et al: International staging system for multiple myeloma. J Clin Oncol 23: 3412-3420, 2005.

24. Livak KJ and Schmittgen TD: Analysis of relative gene expression data using real-time quantitative PCR and the 2(-Delta Delta C(T)) method. Methods 25: 402-408, 2001.

25. Murphy SK, Wylie AA, Coveler KJ, Cotter PD, Papenhausen PR, Sutton VR, Shaffer LG and Jirtle RL: Epigenetic detection of human chromosome 14 uniparental disomy. Hum Mutat 22 92-97, 2003.

26. Benetatos L, Dasoula A, Hatzimichael E, Georgiou I, Syrrou M and Bourantas KL: Promoter hypermethylation of the MEG3 (DLK1/MEG3) imprinted gene in multiple myeloma. Clin Lymphoma Myeloma 8: 171-175, 2008.

27. Sigmon J and Larcom LL: The effect of ethidium bromide on mobility of DNA fragments in agarose gel electrophoresis. Electrophoresis 17: 1524-1527, 1996.

28. Robertson KD: DNA methylation and human disease. Nat Rev Genet 6: 597-610, 2005.

29. Schmidt M, Dehne S and Feierabend J: Post-transcriptional mechanisms control catalase synthesis during its light-induced turnover in rye leaves through the availability of the hemin cofactor and reversible changes of the translation efficiency of mRNA. Plant J 31: 601-613, 2002.

30. Zhang X, Rice K, Wang Y, Chen W, Zhong Y, Nakayama Y, Zhou Y and Klibanski A: Maternally expressed gene 3 (MEG3) noncoding ribonucleic acid: Isoform structure, expression, and functions. Endocrinology 151: 939-947, 2010.
31. Jin L, Cai Q, Wang S, Wang S, Mondal T, Wang J and Quan Z: Long noncoding RNA MEG3 regulates LATS2 by promoting the ubiquitination of EZH2 and inhibits proliferation and invasion in gallbladder cancer. Cell Death Dis 9: 1017, 2018.

32. Chak WP, Lung RW, Tong JH, Chan SY, Lun SW, Tsao SW, Lo KW and To KF: Downregulation of long non-coding RNA MEG3 in nasopharyngeal carcinoma. Mol Carcinog 56: 1041-1054, 2017

33. Muller PA and Vousden KH: Mutant p53 in cancer: New functions and therapeutic opportunities. Cancer Cell 25: 304-317, 2014.

34. Chng WJ, Price-Troska T, Gonzalez-Paz N, Van Wier S, Jacobus S, Blood E, Henderson K, Oken M, Van Ness B, Greipp P, et al: Clinical significance of TP53 mutation in myeloma. Leukemia 21: 582-584, 2007.

35. Drach J, Ackermann J, Fritz E, Krömer E, Schuster R, Gisslinger H, DeSantis M, Zojer N, Fiegl M, Roka S, et al: Presence of a p53 gene deletion in patients with multiple myeloma predicts for short survival after conventional-dose chemotherapy. Blood 92: 802-809, 1998

36. Brooks CL and Gu W: p53 regulation by ubiquitin. FEBS Lett 585: 2803-2809, 2011.

37. Harris SL and Levine AJ: The p53 pathway: Positive and negative feedback loops. Oncogene 24: 2899-2908, 2005.

38. Lu KH, Li W, Liu XH, Sun M, Zhang ML, Wu WQ, Xie WP and Hou YY: Long non-coding RNA MEG3 inhibits NSCLC cells proliferation and induces apoptosis by affecting p53 expression. BMC Cancer 13: 461, 2013.

39. Zhou Y, Zhong Y, Wang Y, Zhang X, Batista DL, Gejman R, Ansell PJ, Zhao J, Weng C and Klibanski A: Activation of p53 by MEG3 non-coding RNA. J Biol Chem 282: 24731-24742, 2007.

40. Feng SQ, Zhang XY, Fan HT, Sun QJ and Zhang M: Upregulation of LncRNA MEG3 inhibits cell migration and invasion and enhances cisplatin chemosensitivity in bladder cancer cells. Neoplasma 65: 925-932, 2018.

41. Zhu J, Liu S, Ye F, Shen Y, Tie Y, Zhu J, Wei L, Jin Y, Fu H, Wu Y and Zheng X: Long noncoding RNA MEG3 interacts with p53 protein and regulates partial p53 target genes in hepatoma cells. PLoS One 10: e0139790, 2015.

42. Zhou Y, Zhang X and Klibanski A: MEG3 noncoding RNA: A tumor suppressor. J Mol Endocrinol 48: R45-53, 2012. 